\title{
Journal of ELECTROSTATICS
}

\section{Liquid dielectrophoresis on the microscale}

\author{
T.B. Jones* \\ Department of Electrical and Computer Engineering, University of Rochester, P.O. Box 270126, Rochester, \\ NY 14627-0126, USA
}

\begin{abstract}
Polarizable liquids respond to a non-uniform electric field by collecting preferentially in regions of maximum field intensity. This manifestation of dielectrophoresis (DEP) is a wellknown example of the ponderomotive force, with the field acting on individual (molecular) dipoles in the liquid. When electrode dimensions are scaled down to $\sim 10$ to $100 \mu \mathrm{m}$, liquid DEP makes possible a new type of high-speed, microfluidic liquid actuator. Of particular interest is that these devices can operate successfully with water-based media which are conductive. DEP microactuation is very fast; nanoliter droplets form in $\sim 0.1 \mathrm{~s}$ and transient velocities of liquid menisci exceed $5 \mathrm{~cm} / \mathrm{s}$. Joule heating is a problem, though proper electrode design and an operational mode where voltage is applied for very short time periods effectively controls temperature rise. The transient flows that occur when voltage is applied are not well understood. Capillarity strongly influences the hydrostatics and dynamics, and surface wetting, which is affected by $\mathrm{rf}$ discharges on the dielectric surface, is also important. (C) 2001 Elsevier Science B.V. All rights reserved.
\end{abstract}

Keywords: Dielectrophoresis; Microfluidics; "Laboratory on a chip"; Capillarity; Liquid actuation; Bernoulli equation

\section{Introduction}

Dielectrophoresis (DEP) is an example of the ponderomotive effect, the basis of which is the force exerted on dipoles by a non-uniform electric field. The phenomenology of liquid DEP may be simply stated: dielectric liquid in a non-uniform electric field tends to collect in regions of high electric field intensity (Fig. 1a). This same force repels a gas or vapor bubble within this liquid from strong fields (Fig. 1b). In these

\footnotetext{
*Tel.: + 1-716-275-5233; fax: + 1-716-473-0486.

E-mail address: jones@ece.rochester.edu (T.B. Jones).
} 


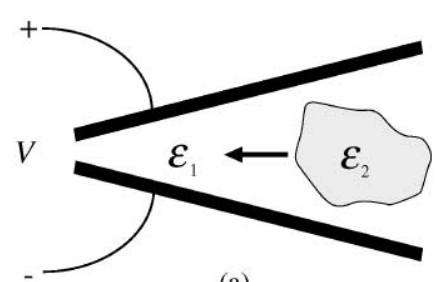

(a)

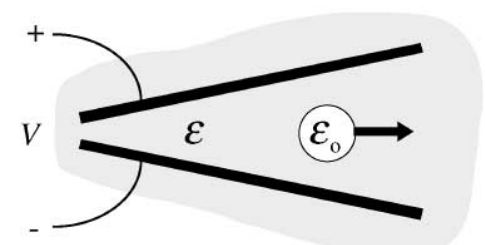

(b)

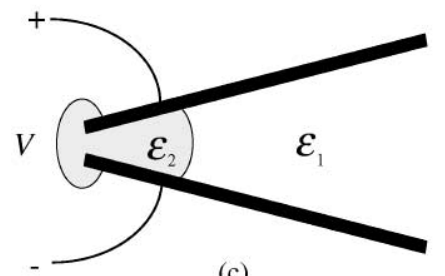

(c)

Fig. 1. Liquid DEP phenomenology: (a) dielectric liquid drawn into strong electric field; (b) bubble repelled from strong electric field; (c) controlled liquid profile with surface parallel to applied electric field.

respects, liquid DEP is the same as particulate dielectrophoresis, as defined by Pohl [1]; however, because they are deformable, liquids also are shaped by the non-uniform electric field. Once drawn into a strong field region, a liquid mass tends to assume a shape such that its free surfaces are approximately parallel to electric field lines (see Fig. 1c). The parallel configuration is more commonly observed because a perpendicular electric field always has a destabilizing influence on plane, liquid/air boundaries [2].

In this paper, the fundamentals of liquid DEP are reviewed and then exemplified by considering the case of sub-microliter volumes of water under the influence of non-uniform rf electric fields created by co-planar microelectrode structures. The distinguishing features of microDEP are identified and possible applications in microfluidics technology are considered.

\section{Liquid DEP fundamentals}

Liquid DEP differs from the ion-drag effect, electroconvection, electro-osmosis, and other electrohydrodynamic (EHD) phenomena in that it is not really a pumping 


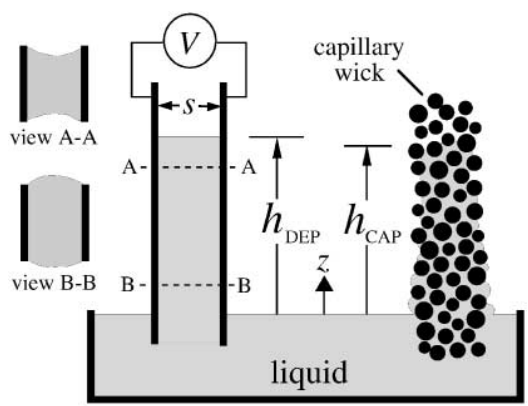

(a)

(b)

Fig. 2. DEP and capillary hydrostatic equilibria. (a) Pellat's original experiment with parallel electrodes [3]. Insets show how liquid profile varies with height $z$. (b) A capillary wick showing how liquid bulges out more at the bottom.

mechanism. Instead, it influences hydrostatic equilibria in a way analogous to capillarity. Fig. 2a shows Pellat's classic experiment [3], consisting of two plane, parallel electrodes at spacing $s$, oriented vertically and partially immersed in a pool of dielectric liquid of mass density $\rho$ and permittivity $\varepsilon$. Gas of negligible density and permeability equal to free space, $\varepsilon_{0}=8.854 \times 10^{-12} \mathrm{~F} / \mathrm{m}$, covers the liquid. The static dielectric height of rise $h_{\mathrm{DEP}}$ is

$$
h_{\mathrm{DEP}} \approx\left(\varepsilon-\varepsilon_{0}\right) E^{2} / 2 \rho g,
$$

where $E \approx V / s$ is the uniform electric field between the plates, $V$ the applied voltage, and $g=9.81 \mathrm{~m} / \mathrm{s}^{2}$ the terrestrial acceleration due to gravity. Pellat's experiment can be understood by comparing it to the static, capillary height of rise of liquid in a wick as depicted in Fig. 2b:

$$
h_{\mathrm{CAP}} \approx 2 \gamma / \rho g r_{\mathrm{c}},
$$

where $\gamma$ is the interfacial surface tension and $r_{\mathrm{c}}$ the capillary pore size. Neither dielectrophoresis nor capillarity can add time-average power to create steady flow of a liquid.

The dielectric siphon is a good example of how a flow can be achieved with DEP [4]. In simple form, this device consists of two closely spaced, facing electrodes running between an upper and a lower reservoir (see Fig. 3). If the voltage is high enough to raise liquid to height $h_{\mathrm{u}}$ from the upper reservoir to the top of the structure, gravity initiates flow. The minimum voltage for siphon action is

$$
V_{\min }=\sqrt{2 \rho g h_{\mathrm{u}} s^{2} /\left(\varepsilon-\varepsilon_{0}\right)} .
$$

If $V<V_{\min }$, liquid drains from the top and flow ceases. Similar to the capillary force in a wick, the DEP force does not pump the liquid in a DEP flow structure, but rather maintains pressure communication between the reservoirs by confining liquid between the electrodes. 


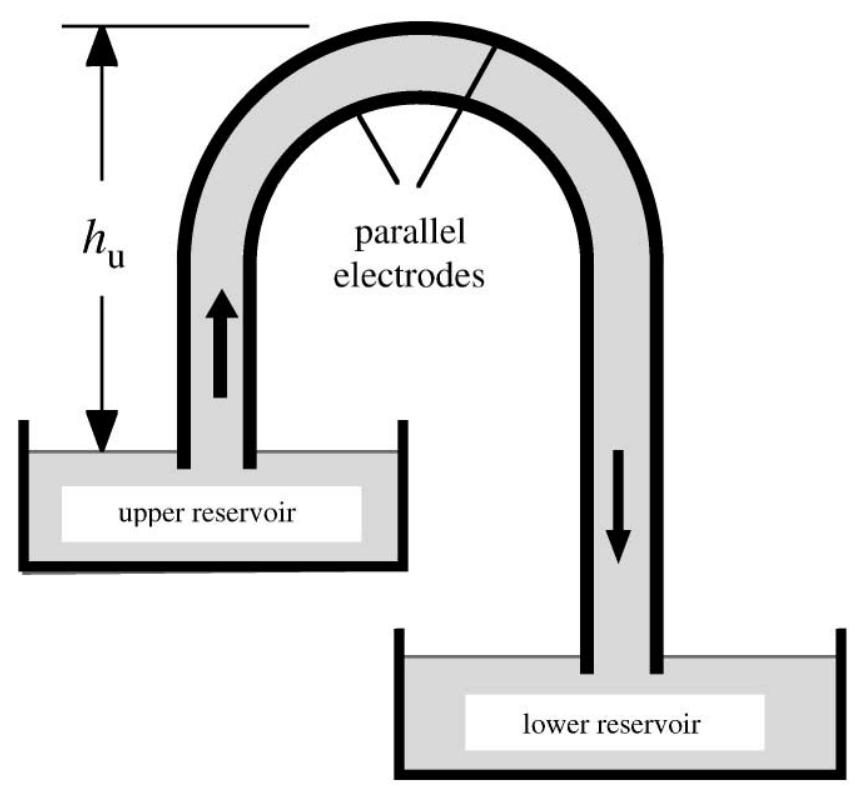

Fig. 3. A simple dielectric siphon consisting of two facing electrodes connecting upper and lower reservoirs.

\section{DEP theory}

The starting point for the theory of liquid DEP is usually the Kelvin-Helmholtz $(\mathrm{K}-\mathrm{H})$ formulation for the volume force density $[5,6]$,

$$
\bar{f}^{\mathrm{e}}=\rho_{\mathrm{f}} \bar{E}-\frac{1}{2} E^{2} \nabla \varepsilon+\nabla\left(\frac{1}{2} E^{2} \rho \partial \varepsilon / \partial \rho\right),
$$

where $\rho_{\mathrm{f}}$ is volume charge density. The associated Maxwell stress tensor is

$$
T_{m n}^{\mathrm{e}}=\varepsilon E_{m} E_{n}-\frac{1}{2} \delta_{m n} E_{k} E_{k}[\varepsilon-\rho(\partial \varepsilon / \partial \rho)] .
$$

The term involving $\partial \varepsilon / \partial \rho$ represents electrostriction, and, in the case of common liquids, which are virtually incompressible under normal conditions, its influence on hydrostatics may be ignored [7].

If $\rho_{\mathrm{f}}=0$ and if the liquid is homogeneous, the $\mathrm{K}-\mathrm{H}$ formulation places the DEP force at interfaces, and it becomes convenient to use the Bernoulli equation to model DEP hydrostatics.

$$
\nabla[p+\rho g z]-\frac{1}{2} E^{2} \nabla \varepsilon=0 .
$$

Here, $p$ is hydrostatic pressure and $z$ is vertical elevation. Within the liquid, the term $p+\rho g z$ is a constant, but at interfaces, the apparent DEP surface force can support hydrostatic pressure discontinuities, much like capillarity does at a curved interface. If the electric field at the surface is tangential and has value $E_{\mathrm{t}}$, the pressure difference is

$$
p_{\text {liquid }}-p_{\text {air }}=\left.T_{z z}^{\mathrm{e}}\right|_{\text {liquid }}-\left.T_{z z}^{\mathrm{e}}\right|_{\text {air }}=-\frac{1}{2}\left(\varepsilon-\varepsilon_{\mathrm{o}}\right) E_{\mathrm{t}}^{2} .
$$


Eq. (1) may be derived using Eq. (6) inside the liquid and Eq. (7) at interfaces inside and outside the electrodes.

Despite the fact that no obvious reference to electric field non-uniformity is invoked in the above derivation, it would be erroneous to conclude that electric field nonuniformity is unnecessary for DEP liquid actuation. The force density $\bar{f}^{\mathrm{e}}$ is derived using an energy argument; strictly speaking, it can only be used to calculate the integrated, total force on an object. Seen in this light, it is clear that DEP liquid actuation in fact does require field non-uniformity. Furthermore, detailed knowledge of the non-uniform fringing field is essential to describe the profile of liquid along the open sides, as shown in Fig. 2a.

\section{Microdielectrophoresis}

With decreasing electrode dimensions, electrostatic forces in general and the DEP force in particular become relatively stronger. If the dimensions of an electrode structure are scaled down by some factor $\alpha<1$, the voltage and electric field strength required to maintain fixed DEP force per unit volume go down by factors of $\alpha^{3 / 2}$ and $\alpha^{1 / 2}$, respectively [8]. Possibly the first demonstration of this favorable scaling was a simple experiment with miniature electrodes fabricated from vapor-deposited Alon-glass [9] (see Fig. 4). Using highly insulating transformer oil $\left(\varepsilon / \varepsilon_{0} \approx 2.4\right.$, $\rho \approx 900 \mathrm{~kg} / \mathrm{m}^{3}$ ) and voltage $V \approx 200 \mathrm{~V}-\mathrm{rms}$ at $60 \mathrm{~Hz}$ ac, the liquid was observed to
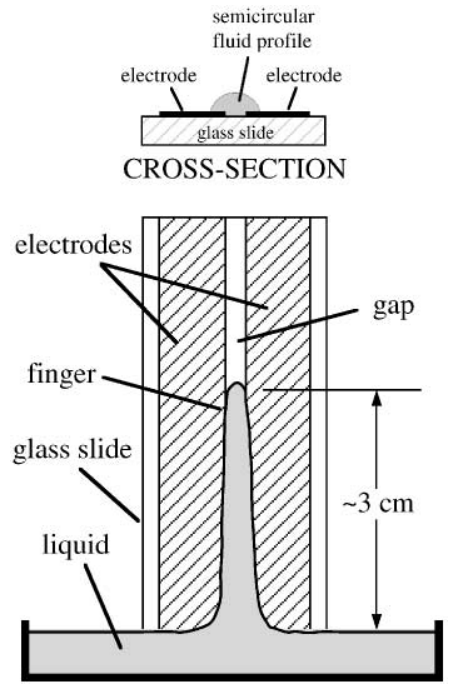

Fig. 4. Early micro DEP experiment with highly insulating transformer oil using co-planar microelectrodes. The liquid finger extends upward in the gap between the electrodes and assumes a compact, semi-circular cross-section. 


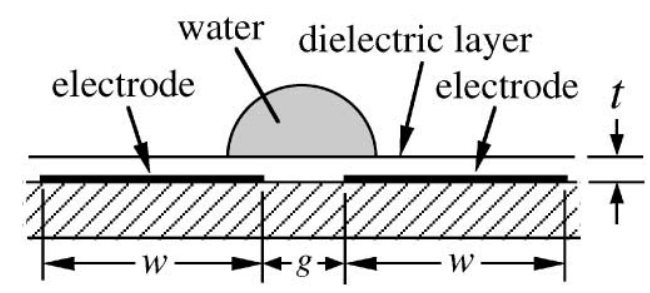

Fig. 5. DEP "wall-less" microstructure with thin, insulating dielectric layer covering the electrodes for use with water. The compact, semicircular profile shown here is observed for $f \geqslant 6 \times 10^{4} \mathrm{~Hz}$.

rise slowly in a narrow finger to a height of several centimeters. The liquid drained very slowly upon removal of the voltage, leaving a thin film.

While this experiment certainly demonstrated the advantages of DEP on the microscale, it attracted little notice because the liquids used were insulating dielectrics. Microfluidic schemes will only make an impact in $\mu$ TAS technology if they can be made to work with aqueous liquids. For DEP actuation of water, the challenge stems from electrolysis and Joule heating. Coating the electrodes with an insulating layer avoids electrolysis. The electrodes used in the experimental work are coplanar strips photolithographically patterned in vapor-deposited Al on glass substrates (see Fig. 5). The dielectric coating is a spin-coated and baked polyimide layer of thickness $t \sim 10 \mu \mathrm{m}$ and dielectric permittivity $\varepsilon_{\mathrm{d}} / \varepsilon_{0} \sim 3$. Fig. 6 shows selected video micrographs of a typical experiment where a $\sim 10 \mu \mathrm{l}$ droplet is deposited on the substrate with a micropipette and then voltage at $\sim 10^{5} \mathrm{~Hz}$ is turned up slowly by hand to $\sim 700 \mathrm{~V}$-rms. A finger projects from the droplet and moves rapidly along the flow structure to the other end. If the voltage is rapidly applied, the finger travels the entire $\sim 3 \mathrm{~cm}$ length of this particular structure in less than $\sim 0.2 \mathrm{~s}$. The transient mass transfer rate is estimated at $\sim 1 \mu \mathrm{l} / \mathrm{s}\left(\sim 10^{-9} \mathrm{~m}^{3} / \mathrm{s}\right)$.

The series capacitance of the dielectric layer dictates use of voltage in the rf range. For frequencies greater than $\sim 60 \mathrm{kHz}$, the water accumulates in the compact, semi-circular cross-section of Fig. 5. Below this limit, the water spreads out slowly in a thin layer and DEP actuation is not achieved. The critical frequency has been estimated successfully from a simple RC circuit model using known electrical parameters for water and the dimensions of the electrodes [10]. This frequency dependence distinguishes liquid DEP from electrowetting, which occurs at much lower frequency [11]. While electrowetting may influence initial formation of the water finger, it has no effect on the liquid profile.

\section{Joule heating}

The strong electric fields needed for actuation and the conductivity of water mean that significant Joule heating can occur. Heat generated within a droplet dissipates by thermal conduction through its area of contact with the substrate, which means that 

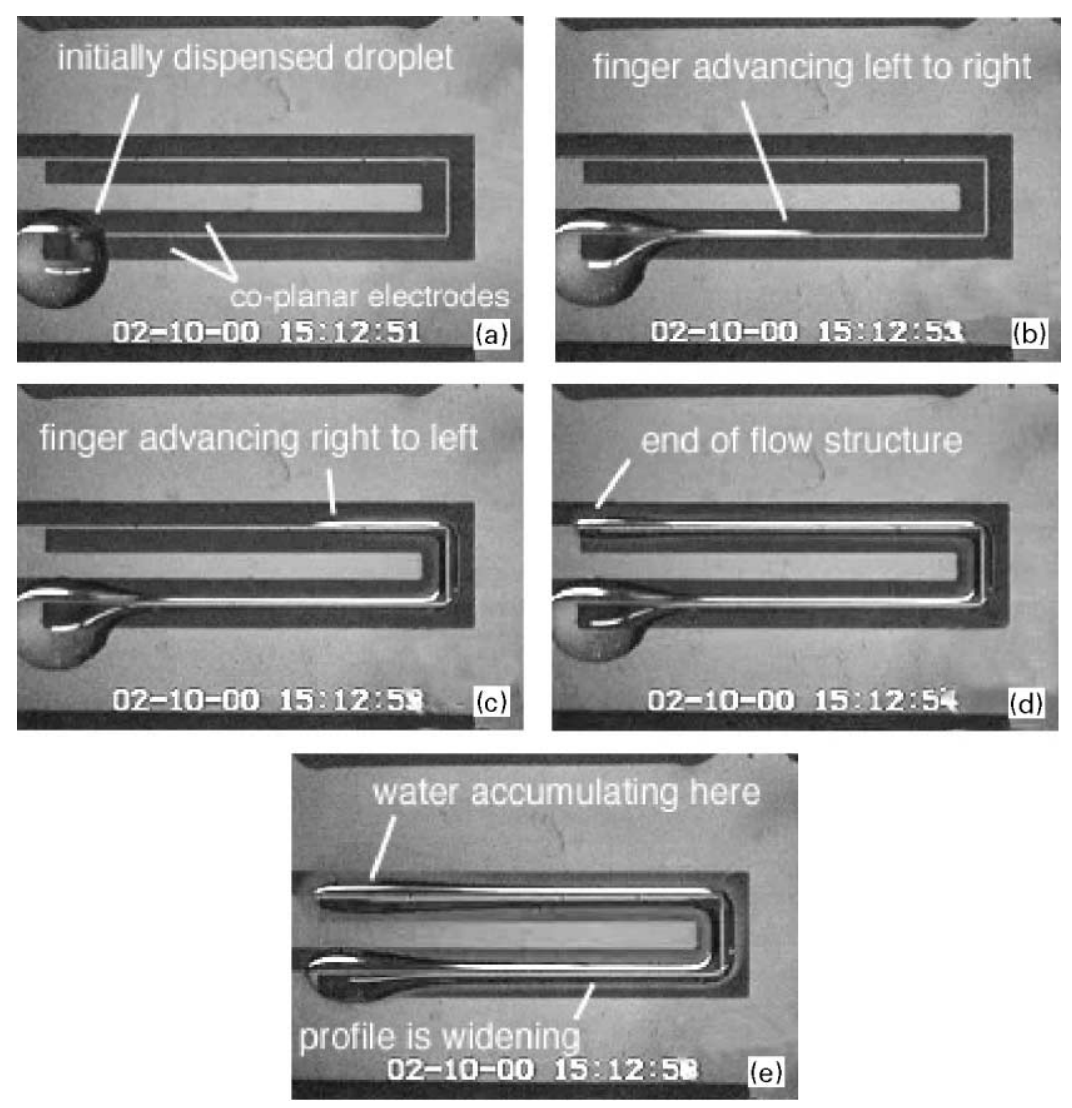

Fig. 6. Sequence of videomicrographs of horizontally mounted flow structure operating with water of electrical conductivity $\sim 10^{-4} \mathrm{~S} / \mathrm{m}, w \approx 1000 \mu \mathrm{m}$, gap $g \approx 100 \mu \mathrm{m}$ rf voltage $\left(\sim 10^{5} \mathrm{~Hz}\right)$ is turned up by hand over a period of several seconds to $\sim 700 \mathrm{~V}$-rms. (a) Voltage off: $\sim 10 \mathrm{ml}$ droplet dispensed by micro-pipette at lower left, (b) voltage on: finger starting to extend to right along flow structure, (c) voltage on: water finger has rounded two $90^{\circ}$ turns, (d) voltage on: water finger has reached end of structure, (e) voltage on: water is now accumulating at end of structure (upper left).

the surface-to-volume ratio controls heat rejection. Therefore, scaling down electrode dimensions and operating with smaller water volumes will reduce heating. In structures like that shown in Fig. 6, evaporation is noticeable if the voltage is left on for more than $\sim 1 \mathrm{~s}$. Fortunately, we can exploit the surprising speed of DEP actuation to reduce further the Joule heating problem, as explained below.

\section{Smart DEP actuation}

Pellat's original demonstration of static liquid dielectrophoresis [3], as well as the dielectric siphon [4], the EHD heat pipe [12], and other applications, implement 
hydrostatic (or almost static) equilibria with steady applied voltage. Heating is not a problem as long as the liquids are insulating dielectrics. On the other hand, DEP actuation of water cannot be achieved in this way because of the relatively high conductivity of water. Even de-ionized water, with conductivity $\sim 10^{-4} \mathrm{~S} / \mathrm{m}$, heats up rapidly. The most promising strategy for exploiting dielectrophoresis with aqueous media is a high speed, transient flow mode where masses of liquid in the nanoliter to microliter range are manipulated by very short applications of voltage. These brief applications of voltage are combined in programmable sequences with changes of the electrode inter-connections to move small volumes around on a substrate, and to divide, mix, and process them. This scheme both minimizes Joule heating and offers an opportunity for the design of fast and smart microfluidic systems for the "laboratory on a chip".

The DEP droplet generator shown in Fig. 7 provides a good example of high speed, electric-field-mediated transient mass transport on a chip. The electrode structure consists of parallel electrode strips terminating in a bisected circle. For the sequence shown in this figure, the voltage has been applied suddenly by a switch. First, a large droplet is deposited on the substrate (7a). When the voltage is applied (7b), a narrow finger extends from the large droplet to the circular electrode set and fills it in $<0.1 \mathrm{~s}$. Finally, after the voltage has been removed (7c), an isolated $\sim 60 \mu$ droplet remains. The bisected, semi-circular electrode structure serves as the basic building block for more complex structures, including a droplet dispenser that has achieved simultaneous formation of multiple droplets in the $\sim 7 \mathrm{nl}$ size range, also $<0.1 \mathrm{~s}[10]$.
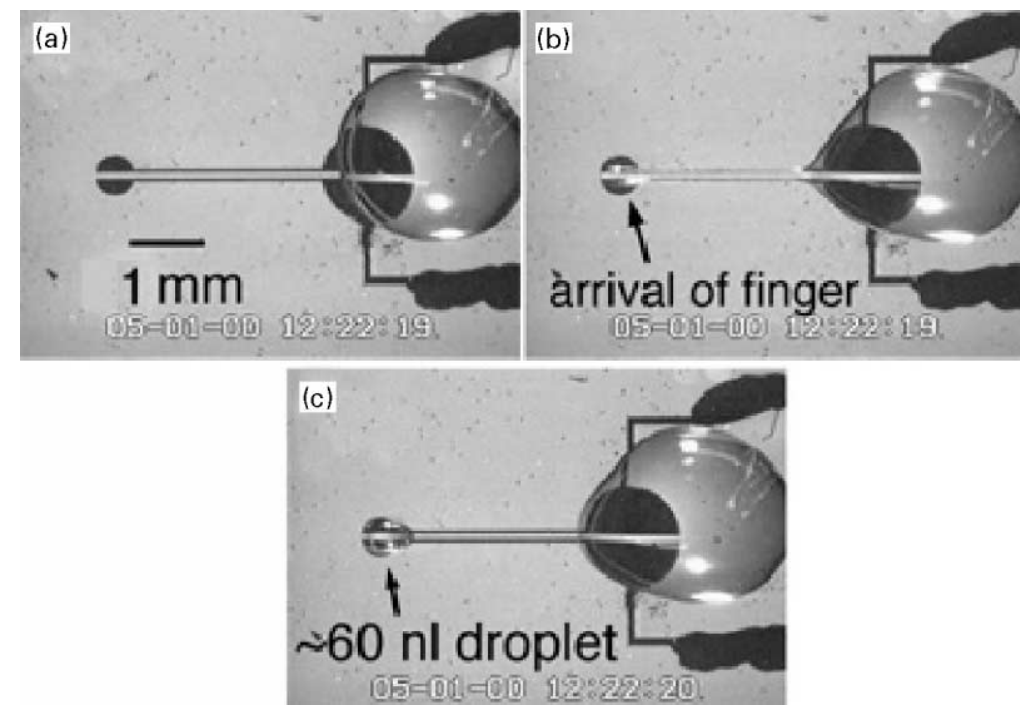

Fig. 7. Planar electrode configuration for droplet formation: (a) large droplet dispensed manually at the right sitting atop the flow structure; (b) with voltage applied, circular electrode pair is covered in $\leqslant 0.1 \mathrm{~s}$; (c) voltage removed, isolated $\sim 60$ nanoliter hemispherical droplet is formed. 

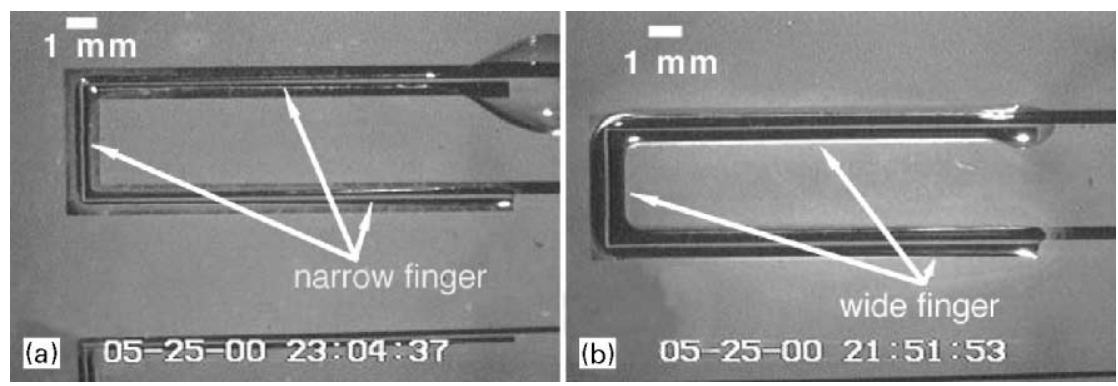

Fig. 8. Indeterminacy of liquid configuration for co-planar electrodes: width $w=500 \mu \mathrm{m}$; gap spacing $g=100 \mu \mathrm{m}$; polyimide layer $t=10 \mu \mathrm{m}$. Both experiments conducted at $\sim 700 \mathrm{~V}$ and $\sim 100 \mathrm{kHz}$. (a) Narrow water finger confined to the gap between electrodes, (b) wide water finger spanning entire width of electrode structure.

\section{Discussion}

One observation that puzzles us is indeterminacy in the width of the liquid finger. Fig. 8 shows micrographs of two experiments with identical electrode structures with electrode spacing $g=100 \mu \mathrm{m}$ and width $w=500 \mu \mathrm{m}$. In Fig. 8a, the finger is confined to the vicinity of the gap between the electrodes, while in Fig. 8b, it spans the entire width of the electrodes. In our experiments, we never observe finger widths intermediate between these two values. One hypothesis to explain this behavior is that intensification of the electric field along the edges of the electrodes provides a strong pinning force that constrains the liquid, holding off the capillary instability that only takes over when voltage is removed [13]. Such a pinning force could constrain the profile in either the wide or narrow configuration. This behavior is observed only for narrower electrode structures and, furthermore, only when $g / w>\sim 0.2$.

At present we are not able to predict which profile will occur in any given experiment, but variable wetting conditions on the surface of the polyimide are certainly influential. There is ample evidence that rf surface discharges alter the surface of the polyimide and in this way affect the advance of the liquid finger along the electrodes. In fact, substrates often work only one time; in subsequent tests, no significant DEP effect can be achieved, even after cleaning and heat treatment. In such cases, it appears that the polyimide surface has become more hydrophilic. Such behavior is no surprise; printing on plastic films routinely uses rf pre-treatment to increase ink acceptance. The discharges we observe along adjacent (inside) edges of the electrodes are quenched when the water finger arrives, and are not the same as those reported by Vallet et al. [14].

\section{Conclusion}

DEP liquid actuation on the microscale provides a means for very rapid manipulation of sub-microliter liquid masses and their transport around a substrate. Nanoliter 
droplet formation in $\sim 0.1 \mathrm{~s}$, transient meniscus speeds exceeding $5 \mathrm{~cm} / \mathrm{s}$, and volume flow rates (also transient) of $\sim 1 \mu \mathrm{l} / \mathrm{s}$ have been obtained. MicroDEP involves a complex interplay of dielectrophoresis, capillarity and surface wetting, transient fluid dynamics, joule heating, and rf discharge phenomena. Many questions must be answered before practical DEP microfluidic devices can be realized. For example, upper limits for transient liquid flow rate and the velocity of water fingers must be established. Also, the lower limit on droplet formation size must be determined. Finally, the very critical upper conductivity limit for effective DEP actuation of aqueous media remains to be investigated. The major challenges to development of a voltage-controlled droplet switchyard include amelioration of Joule heating, scaling down electrode structure sizes to facilitate sub-nanoliter droplet formation, and reduction of the voltage required for liquid actuation. Reducing the thickness of the dielectric coating seems to be the most promising avenue for reducing the voltage requirement and for eliminating $\mathrm{rf}$ discharges. Better materials and improved methods to fabricate dielectric coatings with appropriate surface wetting properties also must be identified.

\section{Acknowledgements}

The author acknowledges the hospitality afforded to him by the Department of Mechanical Engineering of Kyoto University, Japan, during a sabbatical stay during 2000. He is especially grateful to M. Washizu of Kyoto University, whose student, M. Gunji, obtained the videos from which the images shown in Figs. 6,7 and 8 were taken. This research has been supported by long-term fellowships from the Japan Society for the Promotion of Science and from the US National Science Foundation (Center for Global Partnership), and by a grant from the Center for Future Health at the University of Rochester.

\section{References}

[1] H.A. Pohl, J. Appl. Phys. 22 (1951) 869.

[2] J.R. Melcher, Field-Coupled Surface Waves, MIT Press, Cambridge, MA, 1963 (Chapter 3).

[3] H. Pellat, C.R. Seances Acad. Sci. (Paris) 119 (1894) 675.

[4] T.B. Jones, M.P. Perry, J.R. Melcher, Science 174 (1971) 1232.

[5] J.R. Melcher, A Tutorial on Induced Electrohydrodynamic Forces, MIT, Cambridge, MA, 1968.

[6] L.D. Landau, E.M. Lifshitz, in: J.B. Sykes, J.S. Bell (Translators), Electrodynamics of Continuous Media, Pergamon, Oxford, 1960, pp. 64-69.

[7] C.E. Rosenkilde, Proc. R. Soc. (London) A312 (1969) 473.

[8] A.S. Bahaj, A.G. Bailey, Proceedings of the IEEE/IAS Annual Meeting, Cleveland, OH, 1979, pp. $154-157$.

[9] T.B. Jones, unreported experiments, Colorado State University, 1975.

[10] T.B. Jones, M. Gunji, M. Washizu, M.J. Feldman, J. Appl. Phys. 89 (2001) 1441.

[11] J.A.M. Sondag-Huethorst, L.G.L. Fokkink, J. Electroanal. Chem. 367 (1994) 49.

[12] T.B. Jones, Int. J. Heat Mass Transfer 16 (1973) 1045.

[13] S. Schiaffino, A.A. Sonin, J. Fluid Mech. 343 (1997) 95.

[14] M. Vallet, M. Vallade, B. Berge, Eur. Phys. J. B 11 (1999) 583. 